\title{
Time-delayed reaction-diffusion fronts
}

\author{
Neus Isern and Joaquim Fort \\ Departament de Física, Universitat de Girona, 17071 Girona, Catalonia, Spain
}

(Received 22 July 2009; published 20 November 2009)

\begin{abstract}
A time-delayed second-order approximation for the front speed in reaction-dispersion systems was obtained by Fort and Méndez [Phys. Rev. Lett. 82, 867 (1999)]. Here we show that taking proper care of the effect of the time delay on the reactive process yields a different evolution equation and, therefore, an alternate equation for the front speed. We apply the new equation to the Neolithic transition. For this application the new equation yields speeds about $10 \%$ slower than the previous one.
\end{abstract}

DOI: 10.1103/PhysRevE.80.057103

PACS number(s): 89.65.Ef, 87.23.Cc, 89.20.-a

\section{INTRODUCTION}

Reaction-diffusion systems have been applied to many complex biological and physical systems such as population dispersals [1], viral infections [2], chemical reaction processes [3], combustion flames [4], etc. In Ref. [5] a timedelayed model for the front speed was presented including terms up to second order. However, here we will show that there was an error in the mathematical derivation, and we will derive and analyze the behavior of the correct timedelayed equation for the front speed.

In biological systems, variations in the population number density, $p$, are due to two processes: population growth (reproduction minus deaths) and migration (dispersion). The variation due to population growth can be expressed as a Taylor series,

$$
\begin{aligned}
{[p(x, y, t+T)-p(x, y, t)]_{g} } & =\left.T \frac{\partial p}{\partial t}\right|_{g}+\left.\frac{T^{2}}{2} \frac{\partial^{2} p}{\partial t^{2}}\right|_{g}+\cdots \\
& =T F+\left.\frac{T^{2}}{2} \frac{\partial F}{\partial t}\right|_{g}+\cdots
\end{aligned}
$$

where the subindex $g$ denotes growth, we have introduced the growth function as $F(p)=\left.\frac{\partial p}{\partial t}\right|_{g}$, and $T$ is the time delay (one generation in most applications [5]). As usual, we assume that $F(p)>0$.

On the other hand, for the migration (dispersion) we will define the dispersion kernel $\phi\left(\Delta_{x}, \Delta_{y}\right)$ which gives the probability per unit area that an individual initially placed at $(x$ $\left.+\Delta_{x}, y+\Delta_{y}\right)$ has moved to $(x, y)$ after a time interval $T$. Thus, the variation in population number density due to migration can be expressed as [5]

$$
\begin{aligned}
{[p(x, y, t+T)-p(x, y, t)]_{m}=} & \iint p\left(x+\Delta_{x}, y\right. \\
& \left.+\Delta_{y}, t\right) \phi\left(\Delta_{x}, \Delta_{y}\right) d \Delta_{x} d \Delta_{y} \\
& -p(x, y, t) .
\end{aligned}
$$

In a system involving the two processes (population growth and migration), the total variation in population density during a time interval $T$ can be expressed as the sum of both contributions,

$$
\begin{aligned}
p(x, y, t+T)-p(x, y, t)= & \iint p\left(x+\Delta_{x}, y\right. \\
& \left.+\Delta_{y}, t\right) \phi\left(\Delta_{x}, \Delta_{y}\right) d \Delta_{x} d \Delta_{y}-p(x, y, t) \\
& +T F+\left.\frac{T^{2}}{2} \frac{\partial F}{\partial t}\right|_{g}+\cdots .
\end{aligned}
$$

We assume that the kernel is isotropic, i.e., $\phi\left(\Delta_{x}, \Delta_{y}\right)$ $=\phi(\Delta)$, with $\Delta=\sqrt{\Delta_{x}^{2}+\Delta_{y}^{2}}$, and we Taylor expand Eq. (3) up to second order in time and space, thus, obtaining the following reaction-diffusion equation:

$$
\frac{\partial p}{\partial t}+\frac{T}{2} \frac{\partial^{2} p}{\partial t^{2}}=D\left(\frac{\partial^{2} p}{\partial x^{2}}+\frac{\partial^{2} p}{\partial y^{2}}\right)+F+\left.\frac{T}{2} \frac{\partial F}{\partial t}\right|_{g},
$$

where $D$ is the diffusion coefficient $D=\frac{\left\langle\Delta^{2}\right\rangle}{4 T}=\frac{\left\langle\Delta_{x}^{2}\right\rangle}{2 T}=\frac{\left\langle\Delta_{y}^{2}\right\rangle}{2 T}$.

Since $F(p)$ depends only on the population density $p$, then the last term in Eq. (4) can be written as

$$
\left.\frac{T}{2} \frac{\partial F}{\partial t}\right|_{g}=\left.\frac{T}{2} \frac{d F}{d p} \frac{\partial p}{\partial t}\right|_{g}=\frac{T}{2} F^{\prime} F .
$$

In addition, as the density at the leading edge of the front is low, $p \approx 0$, we have that $F(p) \approx p F^{\prime}(0)$ and $F^{\prime}(p) \approx F^{\prime}(0)$. Therefore, for $p \approx 0$ Eq. (4) may be rewritten as

$$
\frac{\partial p}{\partial t}+\frac{T}{2} \frac{\partial^{2} p}{\partial t^{2}}=D\left(\frac{\partial^{2} p}{\partial x^{2}}+\frac{\partial^{2} p}{\partial y^{2}}\right)+p F^{\prime}(0)+\frac{T}{2} p F^{\prime}(0) F(0) .
$$

We now assume that for $t \rightarrow \infty$ and $r \rightarrow \infty$ the front can be considered locally planar. Thus, choosing the $x$ axis parallel to the local speed of the front, $c \equiv\left|c_{x}\right|$, we can look for constant-shape solutions with the form $p=\bar{p} \exp [\lambda(x-c t)]$. Applying this ansatz to Eq. (6) we see that the value of $\lambda$ can be obtained from

$$
\lambda=\frac{-c \pm \sqrt{c^{2}-4\left(D-\frac{T}{2} c^{2}\right) F^{\prime}(0)\left[1+\frac{T}{2} F^{\prime}(0)\right]}}{2\left(D-\frac{T}{2} c^{2}\right)} .
$$

As $\lambda$ has to be real, we obtain a lower bound for the front speed 


$$
c \geq \frac{2 \sqrt{D F^{\prime}(0)\left[1+\frac{T}{2} F^{\prime}(0)\right]}}{1+T F^{\prime}(0)} .
$$

However, the result obtained in Ref. [5] was the so-called HRD speed, namely,

$$
c_{H R D} \geq \frac{2 \sqrt{D F^{\prime}(0)}}{1+\frac{T}{2} F^{\prime}(0)},
$$

which is different from Eq. (8).

The reason for this difference is the following. Here we have used Eq. (5), which allows us to rewrite Eq. (4) as

$$
\frac{\partial p}{\partial t}+\frac{T}{2} \frac{\partial^{2} p}{\partial t^{2}}=D\left(\frac{\partial^{2} p}{\partial x^{2}}+\frac{\partial^{2} p}{\partial y^{2}}\right)+F(p)+\frac{T}{2} \frac{d F}{d p} F .
$$

In contrast, in Ref. [5] the following equation was used:

$$
\frac{\partial p}{\partial t}+\frac{T}{2} \frac{\partial^{2} p}{\partial t^{2}}=D\left(\frac{\partial^{2} p}{\partial x^{2}}+\frac{\partial^{2} p}{\partial y^{2}}\right)+F(p)+\frac{T}{2} \frac{d F}{d p} \frac{\partial p}{\partial t} .
$$

We can see that the last term is different. The reason is that in Ref. [5] the subindex $g$ was omitted in the last term in Eq. (4). Therefore, in Ref. [5], the last term in Eq. (4) was not written as in Eq. (5) but as follows:

$$
\frac{T}{2} \frac{\partial F}{\partial t}=\frac{T}{2} \frac{d F}{d p} \frac{\partial p}{\partial t}=\frac{T}{2} F^{\prime} \frac{\partial p}{\partial t}
$$

and thus leading to Eq. (11) instead of Eq. (10). This is why in Ref. [5], speed (9) was obtained instead of Eq. (8). However, the derivation above clearly shows that Eq. (8) is the right result. In this Brief Report, we will apply variational analysis and show that Eq. (8) is not only a lower bound but the exact speed (Sec. II). We will also analyze the difference between the new Eq. (8) and the HRD speed (9) by applying both equations to the Neolithic transition (Sec. III). In Sec. IV we present our conclusions.

\section{VARIATIONAL ANALYSIS: UPPER BOUND}

Equation (8) is just a lower bound for the speed of front solutions to the new differential equation (4) [or Eq. (10)]. In order to find an upper bound, we apply variational analysis [6] to Eq. (10). As mentioned above, we assume that the fronts have a profile $p(z)=p(x-c t)$ traveling with a speed $c>0$, so all of the derivatives in Eq. (10) can be expressed in terms of $z$. We also assume that the population number density $p>0$ cannot attain values above some value $p_{\max }$, the so-called saturation density. Then, defining $n(p)=-p_{z}$ and assuming that $n(0)=n\left(p_{\max }\right)=0$ and $n>0$ in $\left(0, p_{\max }\right)$, differential equation (10) can be rewritten as

$$
\left(D-c^{2} \frac{T}{2}\right) n \frac{\partial n}{\partial p}-c n+F\left(1+\frac{T}{2} F^{\prime}\right)=0 .
$$

Now, introducing an arbitrary function $g(p)$ such that $g(p)>0$ and $h(p)=-g^{\prime}(p)>0$, we multiply Eq. (13) by $g(p) / n(p)$. Integrating the resulting expression by parts, we obtain

$$
c \int_{0}^{p_{\max }} g d p=\int_{0}^{p_{\max }}\left[\left(D-\frac{T}{2} c^{2}\right) h n+\frac{g}{n} F\left(1+\frac{T}{2} F^{\prime}\right)\right] d p .
$$

Now, we can eliminate $n(p)$ from Eq. (14) applying that for any positive numbers $r$ and $s$, it follows from $(r-s)^{2} \geq 0$ that $(r+s) \geq 2 \sqrt{r s}$. Let us assume that the condition

$$
1+\frac{T}{2} F^{\prime}(p)>0
$$

holds for all $p \in\left(0, p_{\max }\right)$. As $g(p), h(p), n(p), F(p)$, and $\left(D-\frac{T}{2} c^{2}\right)$ are positive [7], we may choose $r \equiv\left(D-\frac{T}{2} c^{2}\right) h n$ and $s \equiv{ }_{n}^{g} F\left(1+\frac{T}{2} F^{\prime}\right)$ into $(r+s) \geq 2 \sqrt{r s}$ and use Eq. (14) to get the following restriction:

$$
\frac{c}{\sqrt{\left(D-\frac{T}{2} c^{2}\right)}} \geq \frac{2 \int_{0}^{p_{\max }} \sqrt{h g F\left(1+\frac{T}{2} F^{\prime}\right)} d p}{\int_{0}^{p_{\max }} g d p} .
$$

Following the method in Ref. [8], Sec. 3.3, it is easy to show that there is a function $g$ for which the equality holds. Then,

$$
\frac{c}{\sqrt{\left(D-\frac{T}{2} c^{2}\right)}}=\max _{g}\left(\frac{2 \int_{0}^{p_{\max }} \sqrt{h g F\left(1+\frac{T}{2} F^{\prime}\right)} d p}{\int_{0}^{p_{\max }} g d p}\right) .
$$

In order to obtain the upper bound for the front speed we will use Jensen's inequality [9]

$$
\frac{\int_{0}^{p_{\max }} \mu(p) \sqrt{\alpha(p)} d p}{\int_{0}^{p_{\max }} \mu(p) d p} \leq \sqrt{\frac{\int_{0}^{p_{\max }} \mu(p) \alpha(p) d p}{\int_{0}^{p_{\max }} \mu(p) d p}},
$$

where $\mu(p)>0$ and $\alpha(p) \geq 0$. We define $\mu(p) \equiv g(p)$ and $\alpha(p) \equiv\left\{h(p) F(p)\left[1+\frac{T}{2} F^{\prime}(p)\right]\right\} / g(p)$. Using these functions into Jensen's inequality (18), and applying the result to Eq. (17), we obtain that

$$
\frac{c}{\sqrt{\left(D-\frac{T}{2} c^{2}\right)}} \leq 2 \max _{g} \sqrt{\frac{\int_{0}^{p_{\max }} h F\left(1+\frac{T}{2} F^{\prime}\right) d p}{\int_{0}^{p_{\max }} g d p}} .
$$

We want an upper bound independent of $g(p)$, so we will first find an expression in which $h(p)=-g^{\prime}(p)$ no longer appears by integrating by parts the numerator in the right-hand side of Eq. (19), 


$$
\int_{0}^{p_{\max }} h F\left(1+\frac{T}{2} F^{\prime}\right) d p=\int_{0}^{p_{\max }} g\left[F^{\prime}\left(1+\frac{T}{2} F^{\prime}\right)+\frac{T}{2} F F^{\prime \prime}\right] d p,
$$

where we have assumed that $F(0)=F\left(p_{\max }\right)=0$ (this holds, for example, for the logistic growth considered in Sec. III).

Moreover, from Eq. (20) we obviously have

$$
\begin{array}{r}
\int_{0}^{p_{\max }} h F\left(1+\frac{T}{2} F^{\prime}\right) d p \leq \\
\sup _{p \in\left(0, p_{\max }\right)}\left[F^{\prime}\left(1+\frac{T}{2} F^{\prime}\right)\right. \\
\left.+\frac{T}{2} F F^{\prime \prime}\right] \int_{0}^{p_{\max }} g d p
\end{array}
$$

so now the upper bound in Eq. (19) is independent of $g(p)$,

$$
\frac{c}{\sqrt{\left(D-\frac{T}{2} c^{2}\right)}} \leq 2 \sqrt{\sup _{p \in\left(0, p_{\max }\right)}\left[F^{\prime}\left(1+\frac{T}{2} F^{\prime}\right)+\frac{T}{2} F F^{\prime \prime}\right] .}
$$

Let us assume that the population growth function $F(p)$ is a continuous function with $F^{\prime \prime}(p) \leq 0$ and $F(0)=0$ (again these assumptions are true for the logistic growth considered in Sec. III). Then $F^{\prime}(p)$ is a decreasing function for increasing values of $p$. Its maximum value is reached for $p=0$. Thus, using the value $p=0$ in Eq. (22) we obtain that the upper bound for the front speed is

$$
c \leq \frac{2 \sqrt{D F^{\prime}(0)\left[1+\frac{T}{2} F^{\prime}(0)\right]}}{1+T F^{\prime}(0)} .
$$

As the lower bound given by Eq. (8) is the same as upper bound (23), we can predict the speed of front solutions to Eq. (10) without any uncertainty,

$$
c=\frac{2 \sqrt{D F^{\prime}(0)\left[1+\frac{T}{2} F^{\prime}(0)\right]}}{1+T F^{\prime}(0)} .
$$

In contrast, for the HRD Eq. (11), the exact speed was previously shown to be [5]

$$
c_{H R D}=\frac{2 \sqrt{D F^{\prime}(0)}}{1+\frac{T}{2} F^{\prime}(0)} \text {. }
$$

\section{APPLICATION TO THE NEOLITHIC TRANSITION}

In order to compare the predictions from Eqs. (24) and (25), we will apply them to the spread of the Neolithic transition in Europe, because this is the case to which Eq. (25) was initially applied [5]. The Neolithic transition is the change from hunter-gatherer to farming economics. In Europe, it took place as an invasion of agricultural populations from the Southeast, which spread across Europe from 13000 to 5000 years before present [10].

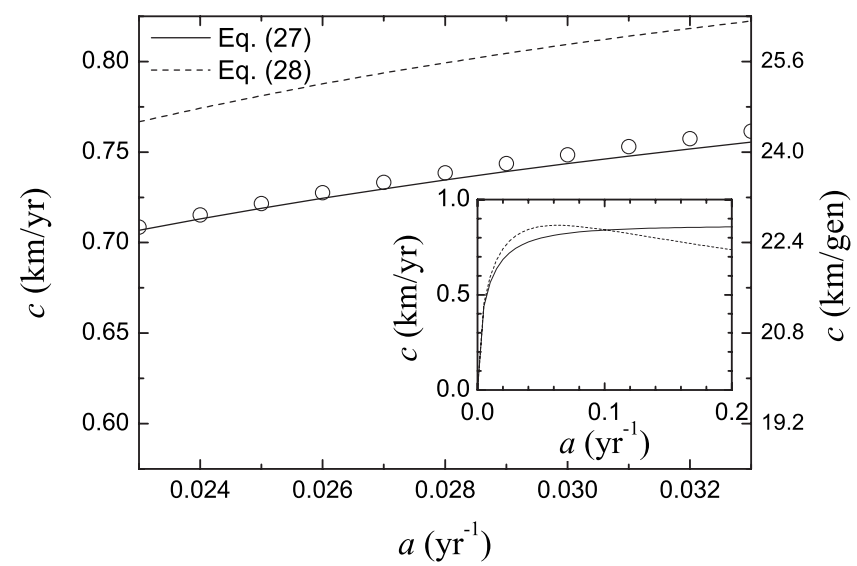

FIG. 1. Comparative plot between the front speed for Eq. (27) (solid line) and Eq. (28) (dashed line). The symbols correspond to the speed obtained from numerically integrating Eq. (10), with $F(p)$ given by Eq. (26). All results have been calculated for a characteristic mobility value $\left\langle\Delta^{2}\right\rangle=1531 \mathrm{~km}^{2}$.

In order to make quantitative predictions we will use the logistic growth function, which has been widely applied to human populations $[5,11]$ :

$$
F(p)=\operatorname{ap}\left(1-\frac{p}{p_{\max }}\right),
$$

where $a$ is called the initial growth rate and $p_{\max }$ is the saturation density.

Using logistic function (26), Eq. (24) can be rewritten as

$$
c=\frac{2 \sqrt{a D\left(1+\frac{a T}{2}\right)}}{1+a T},
$$

whereas the HRD speed (25), used in Ref. [5], is

$$
c_{H R D}=\frac{2 \sqrt{a D}}{1+\frac{a T}{2}} .
$$

Both equations for the front speed depend on three parameters: the initial growth rate, $a$, the diffusion coefficient, $D$ $=\frac{\left\langle\Delta^{2}\right\rangle}{4 T}$, and the generation time, $T$. We will use the ranges $a$ $=0.028 \pm 0.005 \mathrm{yr}^{-1}[12],\left\langle\Delta^{2}\right\rangle=900-2200 \mathrm{~km}^{2}[10]$, and the characteristic value $T=32$ yr [13], which have been measured for preindustrial farming populations. For these ranges, condition (15) is fulfilled, so Eq. (27) gives the speed of fronts.

Figure 1 shows the front speeds obtained from Eqs. (27) and (28) for a characteristic mobility value $\left\langle\Delta^{2}\right\rangle$ $=1531 \mathrm{~km}^{2}$. We can see that, for the range of values for the initial growth rate $a$ appropriate to this application, the new Eq. (27) yields slower speeds than Eq. (28) (about $8 \%$ slower). However, this is not the case for all values of $a$, as can be seen from the inset graph in Fig. 1.

In order to check the validity of Eq. (27), we have also numerically integrated Eq. (10), with $F(p)$ given by Eq. (26), and initially $p=p_{\max }$ in a finite region and $p=0$ elsewhere. 


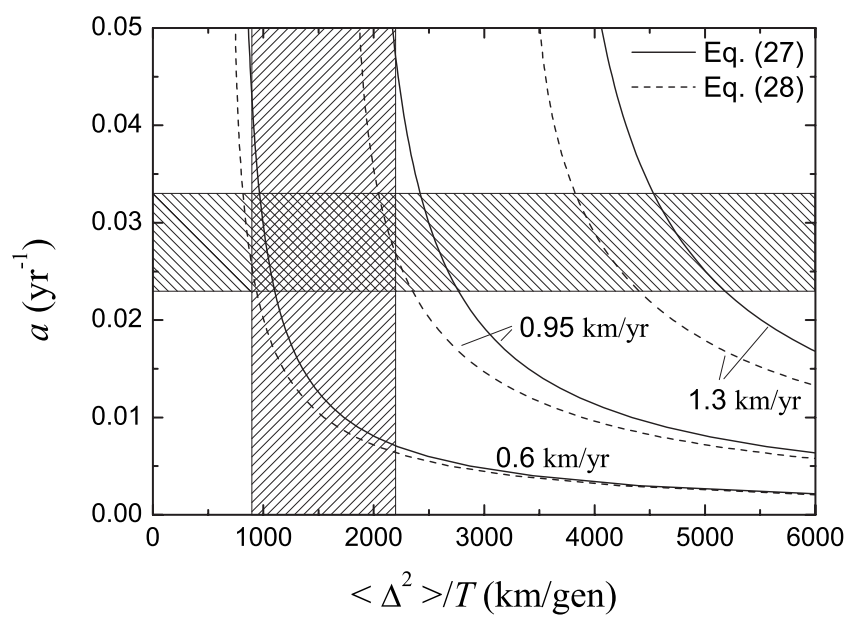

FIG. 2. Predictions for the speed of the wave of advance in the Neolithic transition. The labeled curves correspond to the maximum, minimum, and mean speeds from Neolithic data (0.6-1.3 $\mathrm{km} / \mathrm{yr}$ ). The hatched regions correspond to realistic ranges of the initial growth rate and mobility for the Neolithic transition.

The speed obtained from the numerical integrations corresponds to the circles in Fig. 1. They agree with the new Eq. (27) within less than $0.8 \%$.

The range of speeds for the Neolithic transition front obtained from archeological data is $0.6-1.3 \mathrm{~km} / \mathrm{yr}$ [10]. We can see in Fig. 1 that the results from Eq. (27) lie within this range.

To what extent does our result depend on the uncertainty in the value of the mobility? In Fig. 2, we consider the front speed values $0.6,0.95$, and $1.3 \mathrm{~km} / \mathrm{yr}$, corresponding to the range obtained from archeological data, for Eq. (27) (full lines) and Eq. (28) (dashed lines) [14]. It is seen that the predictions of the new model (full lines) are consistent with the observed front speed for most of the values of the mobility appropriate to this system.

\section{CONCLUDING REMARKS}

In this Brief Report we have improved the derivation of the HRD speed in Ref. [5]. We have obtained the correct evolution Eq. (10) and the new Eq. (24) for the front speed.

We have applied the new Eq. (24) to the Neolithic transition. Using realistic parameters the front speeds are consistent with the observed range for the Neolithic transition in Europe $(0.6-1.3 \mathrm{~km} / \mathrm{yr}$ [10]). Comparing these results with those from the HRD speed, we see that for the Neolithic transition our new equation leads to slower speeds.

In this case, the correction obtained is only about $10 \%$, but it could be higher in other systems where generalizations of Eq. (24) can be useful. For example, our framework could be applied in order to improve the predicted speeds of viral infection fronts [2].

\section{ACKNOWLEDGMENTS}

This work was funded by the European Commission (Grant No. NEST-28192-FEPRE), the MICINN-FEDER (Grant No. FIS2009-13050), and the Generalitat de Catalunya (Grant No. SGR-2009-374). N.I. was supported by the MEC under the FPU program.
[1] K. Davison, P. Dolukhanov, G. R. Sarson, and A. Shukurov, J. Archaeol. Sci. 33, 641 (2006).

[2] J. Fort and V. Méndez, Phys. Rev. Lett. 89, 178101 (2002).

[3] L. Gálfi and Z. Rácz, Phys. Rev. A 38, 3151 (1988).

[4] J. Merikoski, J. Maunuksela, M. Myllys, J. Timonen, and M. J. Alava, Phys. Rev. Lett. 90, 024501 (2003).

[5] J. Fort and V. Méndez, Phys. Rev. Lett. 82, 867 (1999).

[6] R. D. Benguria and M. C. Depassier, Phys. Rev. E 57, 6493 (1998).

[7] The condition $\left(D-T c^{2} / 2\right)>0$ follows from $\lambda<0$ and Eq. (7).

[8] J. Fort and V. Méndez, Rep. Prog. Phys. 65, 895 (2002).

[9] S. Gradshteyn and I. M. Ryzhik, Table of Integrals, Series and Products (Academic, San Diego, 1994); see p. 1133 formula HL151. The inequality is given for convex functions $\phi$. If $\phi$ is a convex function, then $-\phi$ is concave and the inequality for $-\phi$ holds with the sign reversed. Take the function $-\phi$ to be the square root.

[10] R. Pinhasi, J. Fort, and A. J. Ammerman, PLoS Biol. 3, e410 (2005); for the estimation of mobility data see supporting text 3.

[11] J. D. Murray, Mathematical Biology, 3rd ed. (Springer-Verlag, Berlin, 2002), Vol. 1.

[12] N. Isern, J. Fort, and J. Pérez-Losada, J. Stat. Mech.: Theory Exp. (2008) P10012.

[13] J. Fort, D. Jana, and J. Humet, Phys. Rev. E 70, 031913 (2004); for the estimation of the generation time $T=32 \mathrm{yr}$, see note [24] in this reference.

[14] The dashed lines in Fig. 2 are not exactly the same as the lines in Fig. 3 in Ref. [5] because some parameter values have been estimated in a more precise way more recently especially the generation time $T$ [13]. 